\title{
Microstructural alterations in trigeminal neuralgia determined by diffusion tensor imaging are independent of symptom duration, severity, and type of neurovascular conflict
}

\author{
*Juergen Lutz, MD, ${ }^{1}$ Niklas Thon, MD, ${ }^{2}$ Robert Stahl, MD, ${ }^{3}$ Nina Lummel, MD, ${ }^{1}$ \\ Joerg-Christian Tonn, MD, ${ }^{2}$ Jennifer Linn, MD, ${ }^{1}$ and Jan-Hinnerk Mehrkens, MD ${ }^{2}$
}

Departments of ${ }^{1}$ Neuroradiology, ${ }^{2}$ Neurosurgery, and ${ }^{3}$ Radiology, Hospital of the University of Munich, Campus Grosshadern, Munich, Germany

\begin{abstract}
OBJECTIVE In this prospective study diffusion tensor imaging (DTI) was used to evaluate the influence of clinical and anatomical parameters on structural alterations within the fifth cranial nerve in patients with trigeminal neuralgia (TN) due to neurovascular compression.
\end{abstract}

METHODS Overall, 81 patients ( 40 men and 41 women; mean age $60 \pm 5$ years) with typical TN were included who underwent microsurgical decompression. Preoperative 3.0-T high-resolution MRI and DTI were analyzed in a blinded fashion. The respective fractional anisotropy (FA) and apparent diffusion coefficient values were compared with the clinical, imaging, and intraoperative data. This study was approved by the institutional review board, and written informed consent was obtained from all patients.

RESULTS DTI analyses revealed significantly lower FA values within the vulnerable zone of the affected trigeminal nerve compared with the contralateral side $(p=0.05)$. The DTI analyses also included 3 patients without clear evidence of neurovascular conflict on preoperative MRI. No differences were seen between arterial and venous compression. Lower FA values were found 5 months after symptom onset; however, no correlation was found with the duration of symptoms or severity of compression.

CONCLUSIONS DTI analysis allows the quantification of structural alterations, even in those patients without any discernible neurovascular contact on MRI. Moreover, our findings support the hypothesis that both the arteries and veins can cause structural alterations that lead to TN. These aspects can be useful for making treatment decisions.

http://thejns.org/doi/abs/10.3171/2015.2.JNS142587

KEY WORDS trigeminal neuralgia; microvascular decompression; diffusion tensor imaging; neurovascular compression syndrome; diagnostic and operative techniques; pain

$\mathrm{T}$ RIGEMINAL neuralgia (TN) is characterized by recurrent episodes of intense and lancinating pain localized to the sensory supply areas of the fifth cranial nerve $(\mathrm{CN} \mathrm{V})$, which is also known as the trigeminal nerve. ${ }^{11}$ The most frequent cause of TN is mechanical irritation of the nerve within a vulnerable zone that extents for about 4 to $5 \mathrm{~mm}$ from the point of transition from the peripheral (derived from Schwann cells) to the central myelin (derived from the oligodendroglia) (i.e., transition zone) to the most proximal aspect, where the nerve enters the pons (i.e., root entry zone [REZ]). ${ }^{24,25,31,32}$ It is widely believed that direct arterial contact causes pulsatile, repetitive trauma that leads to focal axonal degeneration and demyelination processes within this vulnerable site. ${ }^{9,13,25}$ These structural alterations facilitate ephaptic neurotransmissions that cause pain upon common triggers such as chewing or tactile sensations. ${ }^{11}$ Most frequently, neurovascular compression is caused by a direct anatomical relation with the superior cerebellar artery (SCA) $(60 \%-90 \%)$, followed by the anterior inferior cerebellar

ABBREVIATIONS AD = axial diffusivity; $A D C=$ apparent diffusion coefficient; $A I C A=$ anterior inferior cerebellar artery; $C E=$ contrast enhanced; $C N=$ cranial nerve; $D T I=$ diffusion tensor imaging; FA = fractional anisotropy; FIESTA = fast imaging employing steady-state acquisition; MRA = MR angiography; MVD = microsurgical decompression; $\mathrm{PICA}=$ posterior inferior cerebellar artery; $\mathrm{RD}$ = radial diffusivity; $\mathrm{REZ}$ = root entry zone; $\mathrm{ROI}=$ region of interest; $\mathrm{SCA}=$ superior cerebellar artery; $\mathrm{TN}=$ trigeminal neuralgia.

SUBMITTED November 18, 2014. ACCEPTED February 25, 2015

INCLUDE WHEN CITING Published online September 25, 2015; DOI: 10.3171/2015.2.JNS142587.

* Drs. Lutz and Thon contributed equally to this work. 
artery (AICA) and the basilar artery. ${ }^{11,24,36}$ The relevance of venous contact as a cause for TN remains a matter of debate. ${ }^{5,15,28}$

Currently, high-resolution MRI with steady-state sequences and MR angiography (MRA) are the gold standard for detecting neurovascular conflicts and help to exclude other causes of TN. ${ }^{38}$ These sequences, however, do not allow for objective quantification and prognostication of structural changes that might have occurred in the respective nerve. Diffusion tensor imaging (DTI) allows detailed analysis of white matter integrity by measuring molecular diffusivity. ${ }^{12,27}$ In a previous study, we showed that DTI-including the measurement of its main indices, namely fractional anisotropy (FA) and apparent diffusion coefficient (ADC)-enables the in vivo demonstration of microstructural changes within the affected trigeminal nerve in patients who suffer from a neurovascular compression syndrome. ${ }^{27}$ In the current prospective study, we systematically evaluated FA and ADC values in a large patient cohort that underwent surgery for neurovascular compression-related TN and looked for possible interactions with the clinical and anatomical parameters. In particular, the potential impact of the duration of TN symptoms, the type of vascular contact, and the degree of CN $\mathrm{V}$ compression (as determined by the morphological MRI criteria) on these DTI indices were analyzed. All imaging data were correlated with the intraoperative findings.

\section{Methods}

\section{Patient Population}

Adult patients who underwent microsurgical decompression (MVD) for long-lasting and refractory $\mathrm{TN}$ and for whom both morphological MRI and DTI analyses were available prior to surgery were enrolled between February 2009 and December 2013. All patients were clinically examined by an experienced neurosurgeon (J.-H.M.) and met the criteria of the International Headache Society for TN. ${ }^{11}$ Exclusion criteria included: 1) TN due to other pathological conditions such as multiple sclerosis $(n=12)$, tumors $(n=3)$, or an aneurysm $(n=1) ; 2)$ recurrent disease after previous MVD ( $=17)$; and 3) no DTI analysis before surgery (due to MRI availability or patient-related reasons; $\mathrm{n}=12$ ). All 20 patients included in the initial descriptive study were also considered in the current report. ${ }^{27}$ This study was approved by our institutional review board, and informed consent was obtained from all patients.

\section{MR Imaging Protocol}

MRI was performed on a 3.0-T MRI scanner with an 8-channel head coil (Signa HDx; 3T GE Healthcare) using a standardized protocol..$^{27,33}$ The sequences and their parameters are summarized in Table 1.

\section{Morphological MRI Data Analysis}

Three-dimensional fast imaging employing steadystate acquisition (FIESTA) images and contrast-enhanced (CE) MRA were analyzed collaboratively by 2 experienced neuroradiologists (J.L. and J.L.), who were blinded to the clinical data including the side of nerve affection.
Multiplanar reconstructions were used to assess the trigeminal nerves on both sides separately using a step-bystep approach to determine the presence and severity of the neurovascular conflict, as well as the type of offending vessel and its course with respect to the nerve. Contact was declared if no layer of the CSF was discernible between the nerve and the respective vessel on the high-resolution 3D-FIESTA sequences. The severity of a neurovascular contact was graded using the following system, which was introduced by Sindou et al. for intraoperative grading and adapted for MRI evaluation by Leal et al.:20,35 Grade I indicates that the vessel "touches" the nerve without any visible displacement or distortion of the nerve; Grade II means that the vessel causes displacement and/or distortion of the nerve without indentation on the nerve; and Grade III indicates that the vessel causes a marked indentation on the nerve. If applicable, the vessel (artery or vein) that was responsible for the neurovascular contact was identified on CE-MRA by tracing it back to its parent vessel.

\section{Analysis of the DTI Data Sets}

Postprocessing and analysis of the DTI data sets were performed in a blind fashion (for clinical data and intraoperative findings) using the FuncTool software (release AW4.3_07) on a commercially available workstation (AW 4.2; GE Healthcare). In line with our previous reports, ${ }^{26,27}$ the ADC and FA values were prospectively defined as the primary parameters in the DTI analysis. Accurate measurement was ensured by the same neuroradiologist (J. Lutz) with profound experience using this technique. Special attention was paid to the region of interest (ROI)placement procedure: both high-resolution T2-weighted FIESTA and fused DTI axial sequences were angulated exactly parallel to the cisternal segment of the CN V. FIESTA sequences and a magnification tool (FunkTool software) were used to determine the area of the transition zone in all 81 patients. If necessary, the size of the ellipsoid-shaped ROI was adapted manually to some anatomical variances in order to avoid any confounding bias by inclusion (e.g., CSF or the compressing vascular structure). An example of ROI placement is illustrated in Fig. 1. The software package semiautomatically integrated a motion and eddy current correction step into the postprocessing algorithm. The minimum, mean, and maximum values, as well as the standard deviation of the anisotropy indices (ADC and FA), were calculated and compared with the contralateral side.

\section{Surgery and Outcomes}

Besides clinical evaluation and cranial MRI, the routine preoperative workup included high-resolution CT of the skull base, assessment of acoustic evoked potentials, and an ear, nose, and throat workup. All surgeries were performed by senior neurosurgeons (J.-H.M. or J.-C.T.) with long-standing experience in MVD who were blinded to the DTI data. MVD was performed in the park-bench position via a retromastoid suboccipital craniotomy in all cases. The type of offending vessel, the site of nerve compression, and the results of intraoperative monitoring were documented in each case. Microsurgical decompression 
TABLE 1. Sequence parameters for anatomical imaging

\begin{tabular}{|c|c|c|c|c|c|c|}
\hline Parameter & FIESTA & CE-MRA* & FLAIR & T2-Weighted PROPELLER & FSPGR & DTI \\
\hline TR (msec) & 4.5 & 6.6 & 85 & 51 & 3.2 & 10.5 \\
\hline TE (msec) & 1.8 & 2.3 & 1.2 & 7.5 & 7.9 & 6.5 \\
\hline FOV $(\mathrm{mm})$ & 160 & 180 & 220 & 200 & 220 & 220 \\
\hline Matrix $(\mathrm{mm} \times \mathrm{mm})$ & $256 \times 256$ & $450 \times 450$ & $512 \times 512$ & $480 \times 480$ & $256 \times 256$ & $128 \times 128$ \\
\hline Slice thickness $(\mathrm{mm})$ & 0.6 & 0.8 & 5.0 & 3.0 & 1.0 & 2.0 \\
\hline $\operatorname{NEX}(n)$ & 2 & 1.0 & 1.0 & 1.5 & 1.0 & 4 \\
\hline Resolution & $0.6 \times 0.6 \times 0.6$ & $0.4 \times 0.4 \times 0.6$ & $0.69 \times 0.69 \times 5$ & $0.4 \times 0.4 \times 0.3$ & $1.0 \times 1.0 \times 1.0$ & $1.7 \times 1.7 \times 2.0$ \\
\hline Duration & 7:15 & $7: 15$ & $3: 49$ & $1: 59$ & $3: 42$ & $11: 55$ \\
\hline
\end{tabular}

FLAIR = fluid-attenuated inversion recovery; FSPGR = fast spoiled gradient recalled pulse; NEX = number of excitations; PROPELLER = periodically rotated overlapping parallel lines with enhanced reconstruction.

* CE-MRA was performed first and started 30 seconds after the application of $0.1 \mathrm{mmol} / \mathrm{kg}(0.2 \mathrm{ml} / \mathrm{kg})$ gadobenate dimeglumine (MultiHance; Bracco).

was performed by gentle mobilization of the neurovascular contact and the subsequent insertion of a suitably-sized Teflon sponge (C. R. Bard, Inc.) in order to separate the vessel from the nerve. This procedure was performed in case of either an arterial or venous neurovascular conflict. Intraoperative photographs were stored and made available for subsequent analyses of all patients. Outcomes after surgery were assessed at discharge (around postoperative Day 8) and at a routine follow-up evaluation at 6 to 8 weeks; any peri- and/or postoperative complications were noted.

\section{Statistical Analysis}

Patient, clinical, imaging, and intraoperative data were collected for the entire study population (Tables 1 and 2). In particular, the duration of the clinical symptoms, the type of offending vessel (vein and arteries, separately), and the severity of compression on MRI were compared with the DTI metrics (i.e., FA and ADC). DTI data and the duration of the symptoms were initially assessed for normality using the Kolmogorov-Smirnov test. Parametric clinical data were compared between the left and right sides within the same patient using the 2-tailed Student t-test, and nonnormally distributed data were compared using the Wilcoxon tests. To determine the correlation between the duration of symptoms and the FA and ADC values, the Pearson correlation was calculated. For comparison of differences in FA and ADC values depending on the affecting vessel (artery vs vein), the Spearman test was applied, and to compare differences in different arteries the Mann-Whitney U-test or Wilcoxon test was applied. In this study, $\mathrm{p}$ values $<0.05$ were considered to indicate a statistically significant difference. Data are presented as the mean \pm SD. All statistical calculations were performed using SPSS (version 14).

\section{Results}

\section{Clinical Patient Characteristics}

Overall, 81 patients were enrolled (40 men and 41 women) (Table 2). The mean duration of TN symptoms was $156 \pm 7$ months (range 5-252 months). All patients had TN despite being on medication for at least 5 months before surgery was scheduled. The left side was affected in 30 patients, and the right side was affected in 51 patients. Twenty-four patients presented with an isolated affection in a single branch of the trigeminal nerve, which included the second branch (V2) in 9 patients and the third branch (V3) in 15 patients. No patient exhibited involvement of the first trigeminal branch (V1) alone. Seventeen patients presented with a combined affection of V1 plus V2. The majority (30 patients) presented with a combination of V2 and V3. In 10 patients, all 3 branches (V1-V3) were involved (Table 2).

\section{Morphological MRI Findings}

Conventional MRI revealed a neurovascular conflict on the affected side in 78 of 81 patients (96\%). The severity of nerve compression was classified as Grade I in 18 patients, Grade II in 41 patients, and Grade III in 19 patients. Compression was caused by an artery in 70 of 78 patients $(89.8 \%)$ and concerned the SCA in 60 patients $(77 \%)$, the AICA in 8 patients (10.2\%), and the posterior inferior cerebellar artery (PICA) in 2 patients $(2.5 \%)$, respectively. A typical example of Grade I compression caused by the SCA is presented in Fig. 2. In 8 patients (10.2\%) Grade I compression was caused by the superior petrosal vein $(7$
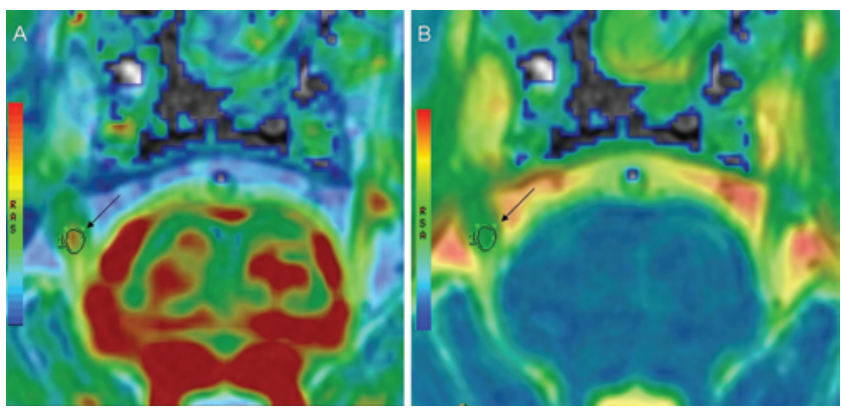

FIG. 1. FIESTA fused with FA (A) and an ADC map (B) of a patient with neurovascular compression and TN affecting the $\mathrm{V} 2$ and $\mathrm{V} 3$ segment on the right side. The ROI (arrow) was manually drawn on the right trigeminal nerve. The color scale of the FA map ranges from 0 to 0.600 , and that of the ADC ranges from 0 to $4.0 \times 10^{-9}$. Figure is available in color online only. 
TABLE 2. Summary of TN patient data

\begin{tabular}{|c|c|}
\hline Factor & Value* $^{*}$ \\
\hline Total & 81 \\
\hline Female & 41 \\
\hline Male & 40 \\
\hline \multicolumn{2}{|c|}{ Symptom duration in mos } \\
\hline Mean & 156 \\
\hline Range & $5-252$ \\
\hline \multicolumn{2}{|l|}{ Side affected } \\
\hline Right & $51(63)$ \\
\hline Left & $30(37)$ \\
\hline \multicolumn{2}{|l|}{ Nerve branch } \\
\hline V1 & $0(0)$ \\
\hline V2 & $9(11)$ \\
\hline V3 & $15(18)$ \\
\hline $\mathrm{V} 1+\mathrm{V} 2$ & $17(21)$ \\
\hline $\mathrm{V} 2+\mathrm{V} 3$ & $30(38)$ \\
\hline V1-3 & $10(12)$ \\
\hline \multicolumn{2}{|l|}{ Vessel† } \\
\hline SCA & $60 / 63(77 / 78)$ \\
\hline AICA & $8 / 8(10.2 / 9.9)$ \\
\hline PICA & $2 / 2(2.5 / 2.5)$ \\
\hline Veins & $8 / 8(10.2 / 9.9)$ \\
\hline SPV & $7 / 7(9 / 8.6)$ \\
\hline TPV & $1 / 1(1.3 / 1.2)$ \\
\hline \multicolumn{2}{|l|}{ Gradeł } \\
\hline 1 & $18(23)$ \\
\hline II & $41(53)$ \\
\hline III & $19(24)$ \\
\hline
\end{tabular}

$\mathrm{SPV}=$ superior petrosal vein; TPV= transverse pontine vein.

* Values are presented as the number of patients (\%) unless otherwise stated. $\dagger 81$ total patients with neurovascular compression; neurovascular compression was visible on MRI for 78 patients, and the remaining 3 revealed SCA contact during surgery. Values are presented as MRI findings/intraoperative findings.

$\ddagger$ Grading system according to Sindou et al.

patients) or the transverse pontine vein (1 patient). In 3 patients (4\%), no direct neurovascular relation was identified on preoperative MRI.

On the clinically unaffected contralateral side, FIESTA MRI revealed neurovascular contact in 14 patients (17\%), all of which were classified as Grade I. The responsible vessel was the SCA in all of these cases $(100 \%)$.

\section{Surgical Results}

The intraoperative findings confirmed the presence of a neurovascular conflict, as well as the type of offending vessel, in each of the 78 cases with MRI-positive findings. A typical example is illustrated in Fig. 2. Additionally, surgery clearly demonstrated contact between the ipsilateral SCA and the affected trigeminal nerve in the 3 remaining patients in whom no such anatomical correlation could be identified on preoperative MRI. Overall, the sensitivity and specificity of the FIESTA images to detect
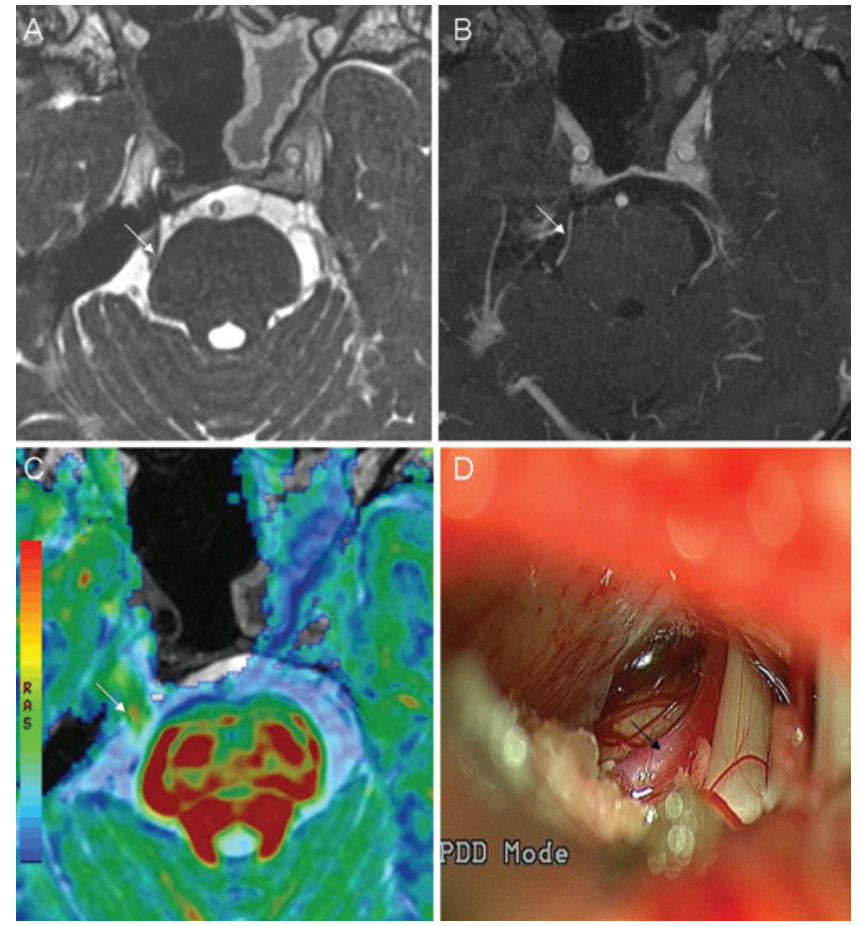

FIG. 2. Axial FIESTA (A), CE-MRA (B), FIESTA fused with the FA map (C), and intraoperative images (D) obtained in a patient with neurovascular compression caused by the SCA (black arrow in D) and TN affecting the $\mathrm{V} 2$ and $\mathrm{V} 3$ segment on the right side. The vessel loop of the right SCA is depicted (white and black arrows) superior to the trigeminal nerve in the MR images, as well as during surgery. Figure is available in color online only.

symptomatic neurovascular compression were $96.3 \%$ and $82.7 \%$, respectively, in our series.

Intraoperatively, the offending vessel crossed the respective nerve superomedially in 49 patients, inferomedially in 18 patients, and superolaterally in 12 patients. In 2 patients, the vessels ran in parallel to the nerve.

Surgery was associated with transient CN VII paresis due to thromboembolic ischemia in 1 patient and with a prolonged stay in the intensive care unit due to early postoperative cerebellar seizures in another case. Facial paresthesia was reported by 4 of 81 patients (4.9\%) directly after surgery with the persistence of symptoms at the 6 -week follow-up reported by 1 of 81 patients $(1.2 \%)$. At discharge, all patients reported the complete relief of TNassociated pain, which was maintained at the first followup evaluation after the withdrawal of all TN medication in 77 of 81 patients (95\%); however, 4 of 81 patients reported some residual pain, which was well controlled by complementary medication.

\section{DTI Findings}

The DTI datasets were always suitable for accurate analyses of the ADC and FA values on both sides. Significant differences in the FA values between the affected and unaffected sides were observed: the mean FA values were significantly lower within the ROI of the clinically affected trigeminal nerve than on the contralateral asymp- 
tomatic side $(0.310 \pm 0.088$ vs $0.384 \pm 0.098 ;$ t-test, $\mathrm{p}=$ 0.004 ) (Fig. 1, Table 3). This was also the case in the 3 patients without identifiable nerve compression on preoperative MRI $(0.321 \pm 0.062$ vs $0.369 \pm 0.091$; t-test, $\mathrm{p}=$ 0.03 ) as well as in the 14 patients with an incidental finding of a neurovascular contact on the contralateral side. More detailed analyses did not reveal significantly different FA values between the arterial $(0.322 \pm 0.034)$ and venous $(0.332 \pm 0.122)$ types of nerve compression, nor the respective offending artery (SCA: $0.316 \pm 0.083$ vs AICA: $0.337 \pm 0.141$ vs PICA: $0.321 \pm 0.054)(\mathrm{t}$-test, all $\mathrm{p}>0.05$ ) Although there was a tendency toward slightly reduced mean FA values of the affected side in cases with more severe compression (Grade I: $0.331 \pm 0.821$ vs Grade II: $0.327 \pm 0.09$ vs Grade III: $0.316 \pm 0.115$ ), these differences did not reach significance (t-test, $\mathrm{p}=0.37$ ). Finally, no significant correlation between FA values and the duration of clinical symptoms was found (Pearson correlation: $r=$ $-0.059 ; \mathrm{p}=0.603$ ).

The corresponding analysis of the mean ADC values regarding the aforementioned clinical and anatomical aspects did not yield any significant result. However, a trend was noted toward higher ADC values for the affected in comparison with the contralateral unaffected trigeminal nerve $\left(2.14 \pm 0.377 \times 10^{-6} \mathrm{~mm}^{2} / \mathrm{sec}\right.$ vs $2.02 \pm 0.392 \times 10^{-6}$ $\mathrm{mm}^{2} / \mathrm{sec}$; t-test, $\mathrm{p}=0.092$ ).

\section{Discussion}

Conventional MRI is currently the gold-standard diagnostic workup for refractory $\mathrm{TN}$ because it offers high-diagnostic accuracy for identifying a neurovascular conflict as the underlying cause. ${ }^{20,21}$ Moreover, the type of offending vessel can be accurately determined. In our study, using CE-MRA data, the respective vessel was ana- tomically and correctly identified by 2 blinded and independent neuroradiological evaluations and matched the intraoperative findings in 78 patients. In 3 patients, however, no neurovascular conflict was discernible on preoperative high-resolution MRI, even when reevaluated in a retrospective fashion. Notably, MVD led to an immediate relief of symptoms in these rare cases as well. Currently, it remains speculative whether dynamic processes such as the positioning of the patient might affect the vascular anatomy within the cerebellopontine cistern and lead to false-negative results on conventional MRI in rare cases.

In accordance with the literature, neurovascular conflict most frequently involved the SCA. ${ }^{20,24,36}$ However, both MRI data and intraoperative findings confirmed a venous conflict as the underlying cause of TN in about $10 \%$ of patients. Both the petrosal vein and the transverse pontine vein have been described in this context before..${ }^{15}$ Notably, MVD led to the complete remission of pain attacks in this subpopulation as well. Overall, the high success rate of treating with MVD in our study population was certainly due to a thorough clinical evaluation with the critical exclusion of those patients with uncertainties concerning the etiology and patients with, for example, multiple sclerosis as the underlying cause of TN. This observation is in line with previous reports..$^{19,37}$

The pathophysiological role of venous conflict is controversially discussed in the literature..$^{15,16,34}$ Currently, it is believed that pulsatile and repetitive mechanical irritations due to direct arterial contact are required and drive the morphological and functional alterations that result in TN: histopathological studies have demonstrated large demyelinated axons,,$^{13,25}$ loss of axon quantity, ${ }^{9}$ and abnormal remyelinization processes within the vulnerable sites of the affected nerve. ${ }^{24,25}$ In agreement with these histological findings, neurosurgeons often describe the affected

TABLE 3. Summary of DTI metrics*

\begin{tabular}{|c|c|c|c|c|c|c|}
\hline \multirow[b]{4}{*}{ Factor } & \multicolumn{5}{|c|}{ FA and ADC Values of the Affected Nerves } & \\
\hline & \multicolumn{6}{|c|}{ DTI Index } \\
\hline & \multicolumn{3}{|c|}{ FA } & \multicolumn{3}{|c|}{$A D C$} \\
\hline & Mean & SD & $\mathrm{p}$ Value & Mean & SD & $\mathrm{p}$ Value \\
\hline Affected side & 0.310 & 0.0876 & \multirow[t]{2}{*}{0.005} & 2.14 & 0.377 & \multirow[t]{2}{*}{0.092} \\
\hline Unaffected side & 0.384 & 0.0977 & & 2.02 & 0.392 & \\
\hline \multicolumn{7}{|l|}{ Vessel } \\
\hline SCA & 0.316 & 0.083 & & 2.05 & 0.361 & \\
\hline AICA & 0.337 & 0.141 & & 2.03 & 0.223 & \\
\hline PICA & 0.321 & 0.054 & & 2.18 & 0.259 & \\
\hline Sum of the arteries & 0.322 & 0.034 & \multirow[t]{2}{*}{0.535} & 2.05 & 0.361 & \multirow[t]{2}{*}{0.767} \\
\hline Sum of the veins & 0.332 & 0.122 & & 2.17 & 0.507 & \\
\hline \multicolumn{7}{|l|}{ Grade } \\
\hline I & $0.331 \dagger$ & 0.821 & & $2.07 \dagger$ & 0.569 & \\
\hline$\|$ & $0.327 \dagger$ & 0.0901 & & $2.15 \dagger$ & 0.375 & \\
\hline III & $0.316 \dagger$ & 0.115 & 0.37 & $2.18 \dagger$ & 0.483 & 0.21 \\
\hline
\end{tabular}


trigeminal nerve as flattened and edematous in situ. Functionally, ephaptic neurotransmissions seem to promote the sensation of pain due to common triggers like chewing or touching. ${ }^{11}$ While some authors argue that only arterial contact can cause the required pulsatile impact, others hypothesize that this phenomenon can also occur in patients with venous contact due to pulsatile flow that is propagated backward by the heart rate in the absence of intravenous valves. ${ }^{2,34}$ Some authors even question the essential role of the pulsatile impact at all.,15,24,36 From the clinical perspective, TN-related symptoms do not differ between patients with arterial or venous neurovascular conflicts. ${ }^{15}$ It remains unknown to what extent structural alterations of the affected nerve might differ depending on the type of offending vessel (arterial or venous). Unfortunately, common imaging protocols cannot be used to visualize structural alterations that are associated with TN. ${ }^{20}$

\section{DTI of TN Due to Neurovascular Compression}

In recent years, several studies demonstrated that DTI is feasible for detecting microstructural alterations in the trigeminal nerve in vivo, and thus seems to be of additional diagnostic value in patients with neurovascular compression-associated TN. ${ }^{8,21,27}$ FA and ADC represent the 2 DTI indices that are most widely used because they reliably indicate abnormalities in white matter tracts, independent of their cause..$^{3,26,30}$ The measurement of FA provides robust values for quantifying the degree of directionality of diffusion (i.e., anisotropic diffusion) as a marker of microstructural integrity. It is generally believed that degeneration of the white matter tracts results in a reduction in FA, as well as in an increase in ADC values, due to diffusivity being averaged in all spatial directions as a result of the loss of myelin and axonal membranes. ${ }^{3}$

FA and ADC values can be reliably determined, even in anatomically small areas such as the vulnerable site of nerve compression in TN patients. ${ }^{8,27}$ It must be ensured, however, that the ROI is accurately placed to avoid any confounding influence (e.g., from the CSF). In our previous study we showed that the FA values were significantly lower within the affected trigeminal nerve in comparison with the contralateral asymptomatic trigeminal nerve. In the current prospective evaluation of a large number of patients, a clear correlation between decreased FA values and symptomatic nerve compression was confirmed. Lower FA values were also seen on the symptomatic side in the 3 patients with negative MRI and in those 14 patients with an incidental finding of a neurovascular contact on the contralateral side; in this subpopulation, the FA values on the contralateral asymptomatic side matched the values that have been described for normal controls, ${ }^{26}$ despite Grade I compression on MRI. The latter aspect is supported by Lin and colleagues who also found regular FA and ADC values in asymptomatic individuals with vascular compression of the trigeminal nerve. ${ }^{22}$ Therefore, DTI analysis provides qualitative information that differs from what can be seen on conventional MRI alone.

\section{Influence of Severity of Compression on FA Values}

With regard to the severity of neurovascular compression, a higher degree of compression has been previously shown to correlate with a decreased volume of the nerve, and lower FA and higher ADC values. ${ }^{20}$ Based on these findings, one might hypothesize that more severe neurovascular compression would result in more severe microstructural changes, indicating linear progressive damage of the nerve with consecutive quantifiable atrophy. Interestingly, no significant correlation between FA values and the severity of neurovascular contact, as assessed by preoperative MRI, was found. This finding might be partially explained by the suggested pathomechanism of pain generation in TN patients. ${ }^{24,31}$ It has been demonstrated in animal studies that the supposed pulsatile nature of pathological vascular contact results in a process of de- and remyelination of the nerve root, rather than progressive demyelination. 2,6 This process, together with an altered pain-processing threshold, is thought to be responsible for the typical paroxysmal and not constant pain syndrome seen in TN. Based on this observation, it can be hypothesized that the observed changes in FA values reflect this process of myelin reorganization, rather than linear progressive demyelination.

\section{Influence of Duration of Compression on FA Values}

Lowered FA values were a robust phenomenon within the affected nerve and were already seen in the TN patient who was scheduled for surgery 5 months after symptom onset; no correlation between the duration of symptoms and FA values was found (Tables 2 and 3). This observation contradicts a linear correlation between the overall symptom duration and the amount of microstructural alterations within the vulnerable zone, as determined by DTI. Moreover, the detection of lowered FA values, even at the early stages of the disease, is well in accordance with previous studies on the time courses of the de- and remyelination processes of experimental cranial nerve compression syndromes: ${ }^{2,6}$ for the optic nerve in cats, it has been shown that demyelination starts as early as 2 days after the onset of mechanical compression and that remyelination with abnormal nerve tissue thickness and organization develop after several weeks. Accordingly, in another animal model of neurovascular compression-related TN, rats developed allodynia on Day 3 after artificial compression of the nerve root with plastic filaments. ${ }^{7}$

\section{Influence of the Type of Offending Vessel on FA Values}

Interestingly, the DTI indices did not differ significantly between patients with arterial and those with a venous compression syndrome, indicating a very similar microstructural alteration of the affected trigeminal nerves independent of the nature of the contact.

\section{FA Analysis, but not ADC, Identifies Structural Alterations}

While differences in the FA values are a consistent finding in all published studies on this issue, the published data regarding ADC values in TN patients yield variable results: some authors found no significant differences, and others report significantly higher ADC values on the affected versus the unaffected side. ${ }^{9,10,23,27}$ Here, no significant correlation between ADC values and any of the clinical, anatomical, and intraoperative findings was seen. This also included patients with a more severe, Grade III 
neurovascular compression. This observation might further indicate that FA analysis is more sensitive to subtle microstructural changes than ADC.12,17

Recent data derived from ex vivo and animal studies using stronger magnetic fields $(>3 \mathrm{~T})$ have shown that 2 DTI parameters-axial diffusivity (AD) and radial diffusivity (RD) - correlate with axonal demyelination ${ }^{18}$ and axonal loss. ${ }^{4}$ These indices are calculated from the principle eigenvectors and take the directionality of the ellipsoid into account, which is averaged in the calculation of the ADC. It has been shown that both AD and RD values might be higher in patients with TN. ${ }^{8}$ This issue, however, remains controversial, as no such correlation was found by Liu et al. who also showed that FA is the most important DTI biomarker when monitoring TN, whereas ADC, AD, and RD are less sensitive. ${ }^{23}$ More data are needed to evaluate new DTI parameters and these particular issues.

\section{Technical Limitations}

All DTI studies on cranial nerves have to cope with the potential partial volume effects caused by the small sizes of the anatomical structures. Together with the optimized protocol, particularly the highly controlled ROI placement procedure that was based on the fusion of the high-resolution FIESTA and DTI sequences, we are confident to have minimized this effect in the trigeminal nerves.

Our current protocol does not enable reliable follow-up DTI analyses after MVD (as it is described after radiosurgery),${ }^{14}$ which most likely is due to the artifacts caused by the surgical procedure itself.

\section{Conclusions}

DTI reveals alterations in the anisotropy index FA within the REZ of the trigeminal nerve in patients with neurovascular compression-related TN. These findings were independent of the duration of symptoms, the severity of compression, and the type of the offending vessel. These findings indicate that FA analysis enables the in vivo visualization of changes in the microstructural integrity that are associated with TN. These alterations seem to be present early in the course of the disease and might reflect an alternating process of de- and remyelinization (i.e., a "remodelling" of the myelin sheaths rather than a constant decline in myelinization). We could demonstrate that pathological DTI indices cannot only be caused by arterial but also venous contact, which strongly supports the hypothesis that both arteries and veins can cause TN and might therefore be equally eligible targets for decompression surgery. FA analysis also indicates structural changes in patients without any discernible neurovascular contact on MRI. This aspect might be particularly useful for supporting the decision to perform surgery in patients with the typical clinical presentation of a neurovascular compression-related TN that fulfills the clinical criteria for MVD, but in whom conventional MRI does not demonstrate a neurovascular conflict. In turn, patients without clinical symptoms but with a neurovascular contact on morphological MRI also showed unsuspicious FA values, which might also be a helpful aspect to consider in future treatment decision making. Further studies should address the correlation between changes in the DTI indices and postoperative outcomes in these patients.

\section{References}

1. Adamczyk M, Bulski T, Sowińska J, Furmanek A, Bekiesińska-Figatowska M: Trigeminal nerve - artery contact in people without trigeminal neuralgia - MR study. Med Sci Monit 13 (Suppl 1):38-43, 2007

2. Apfelbaum RI: Neurovascular decompression: the procedure of choice? Clin Neurosurg 46:473-498, 2000

3. Basser PJ, Pierpaoli C: Microstructural and physiological features of tissues elucidated by quantitative-diffusion-tensor MRI. 1996. J Magn Reson 213:560-570, 2011

4. Budde MD, Xie M, Cross AH, Song SK: Axial diffusivity is the primary correlate of axonal injury in the experimental autoimmune encephalomyelitis spinal cord: a quantitative pixelwise analysis. J Neurosci 29:2805-2813, 2009

5. Chen GQ, Wang XS, Wang L, Zheng JP: Arterial compression of nerve is the primary cause of trigeminal neuralgia. Neurol Sci 35:61-66, 2014

6. Clifford-Jones RE, Landon DN, McDonald WI: Remyelination during optic nerve compression. Trans Ophthalmol Soc U K 100:274-275, 1980

7. Clifford-Jones RE, McDonald WI, Landon DN: Chronic optic nerve compression. An experimental study. Brain 108:241-262, 1985

8. DeSouza DD, Hodaie M, Davis KD: Abnormal trigeminal nerve microstructure and brain white matter in idiopathic trigeminal neuralgia. Pain 155:37-44, 2014

9. Devor M, Govrin-Lippmann R, Rappaport ZH: Mechanism of trigeminal neuralgia: an ultrastructural analysis of trigeminal root specimens obtained during microvascular decompression surgery. J Neurosurg 96:532-543, 2002

10. Fujiwara S, Sasaki M, Wada T, Kudo K, Hirooka R, Ishigaki D, et al: High-resolution diffusion tensor imaging for the detection of diffusion abnormalities in the trigeminal nerves of patients with trigeminal neuralgia caused by neurovascular compression. J Neuroimaging 21:e102-e108, 2011

11. Headache Classification Committee of the International Headache Society: Classification and diagnostic criteria for headache disorders, cranial neuralgias and facial pain. Cephalalgia 8 (Suppl 7):1-96, 1988

12. Herweh C, Kress B, Rasche D, Tronnier V, Tröger J, Sartor $\mathrm{K}$, et al: Loss of anisotropy in trigeminal neuralgia revealed by diffusion tensor imaging. Neurology 68:776-778, 2007

13. Hilton DA, Love S, Gradidge T, Coakham HB: Pathological findings associated with trigeminal neuralgia caused by vascular compression. Neurosurgery 35:299-303, 1994

14. Hodaie M, Chen DQ, Quan J, Laperriere N: Tractography delineates microstructural changes in the trigeminal nerve after focal radiosurgery for trigeminal neuralgia. PLoS One 7:e32745, 2012

15. Hong W, Zheng X, Wu Z, Li X, Wang X, Li Y, et al: Clinical features and surgical treatment of trigeminal neuralgia caused solely by venous compression. Acta Neurochir (Wien) 153:1037-1042, 2011

16. Jannetta PJ: Arterial compression of the trigeminal nerve at the pons in patients with trigeminal neuralgia. 1967. J Neurosurg 107:216-219, 2007

17. Khong PL, Zhou LJ, Ooi GC, Chung BH, Cheung RT, Wong VC: The evaluation of Wallerian degeneration in chronic paediatric middle cerebral artery infarction using diffusion tensor MR imaging. Cerebrovasc Dis 18:240-247, 2004

18. Klawiter EC, Schmidt RE, Trinkaus K, Liang HF, Budde MD, Naismith RT, et al: Radial diffusivity predicts demyelination in ex vivo multiple sclerosis spinal cords. Neuroimage 55:1454-1460, 2011

19. Leal PR, Barbier C, Hermier M, Souza MA, Cristino-Filho 
G, Sindou M: Atrophic changes in the trigeminal nerves of patients with trigeminal neuralgia due to neurovascular compression and their association with the severity of compression and clinical outcomes. J Neurosurg 120:1484-1495, 2014

20. Leal PR, Hermier M, Froment JC, Souza MA, Cristino-Filho G, Sindou M: Preoperative demonstration of the neurovascular compression characteristics with special emphasis on the degree of compression, using high-resolution magnetic resonance imaging: a prospective study, with comparison to surgical findings, in 100 consecutive patients who underwent microvascular decompression for trigeminal neuralgia. Acta Neurochir (Wien) 152:817-825, 2010

21. Leal PR, Roch JA, Hermier M, Souza MA, Cristino-Filho G, Sindou M: Structural abnormalities of the trigeminal root revealed by diffusion tensor imaging in patients with trigeminal neuralgia caused by neurovascular compression: a prospective, double-blind, controlled study. Pain 152:23572364, 2011

22. Lin W, Chen YL, Zhang QW: Vascular compression of the trigeminal nerve in asymptomatic individuals: a voxel-wise analysis of axial and radial diffusivity. Acta Neurochir (Wien) 156:577-580, 2014

23. Liu Y, Li J, Butzkueven H, Duan Y, Zhang M, Shu N, et al: Microstructural abnormalities in the trigeminal nerves of patients with trigeminal neuralgia revealed by multiple diffusion metrics. Eur J Radiol 82:783-786, 2013

24. Love S, Coakham HB: Trigeminal neuralgia: pathology and pathogenesis. Brain 124:2347-2360, 2001

25. Love S, Hilton DA, Coakham HB: Central demyelination of the Vth nerve root in trigeminal neuralgia associated with vascular compression. Brain Pathol 8:1-12, 1998

26. Lummel N, Mehrkens JH, Linn J, Buchholz G, Stahl R, Bochmann K, et al: Diffusion tensor imaging of the trigeminal nerve in patients with trigeminal neuralgia due to multiple sclerosis. Neuroradiology 57:259-267, 2015

27. Lutz J, Linn J, Mehrkens JH, Thon N, Stahl R, Seelos K, et al: Trigeminal neuralgia due to neurovascular compression: high-spatial-resolution diffusion-tensor imaging reveals microstructural neural changes. Radiology 258:524-530, 2011

28. Matsushima T, Huynh-Le P, Miyazono M: Trigeminal neuralgia caused by venous compression. Neurosurgery 55:334-339, 2004

29. Miller JP, Acar F, Hamilton BE, Burchiel KJ: Radiographic evaluation of trigeminal neurovascular compression in patients with and without trigeminal neuralgia. J Neurosurg 110:627-632, 2009

30. Moseley ME, Cohen Y, Kucharczyk J, Mintorovitch J, Asgari HS, Wendland MF, et al: Diffusion-weighted MR imaging of anisotropic water diffusion in cat central nervous system. Radiology 176:439-445, 1990

31. Nurmikko TJ, Eldridge PR: Trigeminal neuralgia-patho- physiology, diagnosis and current treatment. Br J Anaesth 87:117-132, 2001

32. Peker S, Kurtkaya O, Uzün I, Pamir MN: Microanatomy of the central myelin-peripheral myelin transition zone of the trigeminal nerve. Neurosurgery 59:354-359, 2006

33. Pierpaoli C, Basser PJ: Toward a quantitative assessment of diffusion anisotropy. Magn Reson Med 36:893-906, 1996

34. Sekula RF, Frederickson AM, Jannetta PJ, Bhatia S, Quigley MR, Abdel Aziz KM: Microvascular decompression in patients with isolated maxillary division trigeminal neuralgia, with particular attention to venous pathology. Neurosurg Focus 27(5):E10, 2009

35. Sindou M, Leston J, Decullier E, Chapuis F: Microvascular decompression for primary trigeminal neuralgia: longterm effectiveness and prognostic factors in a series of 362 consecutive patients with clear-cut neurovascular conflicts who underwent pure decompression. J Neurosurg 107:11441153,2007

36. Thomas KL, Vilensky JA: The anatomy of vascular compression in trigeminal neuralgia. Clin Anat 27:89-93, 2014

37. Xia L, Zhong J, Zhu J, Wang YN, Dou NN, Liu MX, et al: Effectiveness and safety of microvascular decompression surgery for treatment of trigeminal neuralgia: a systematic review. J Craniofac Surg 25:1413-1417, 2014

38. Yoshino N, Akimoto H, Yamada I, Nagaoka T, Tetsumura A, Kurabayashi T, et al: Trigeminal neuralgia: evaluation of neuralgic manifestation and site of neurovascular compression with 3D CISS MR imaging and MR angiography. Radiology 228:539-545, 2003

\section{Disclosure}

The authors report no conflict of interest concerning the materials or methods used in this study or the findings specified in this paper.

\section{Author Contributions}

Conception and design: Lutz, Thon, Linn, Mehrkens. Acquisition of data: Lutz, Mehrkens. Analysis and interpretation of data: Lutz, Thon, Stahl, Lummel, Mehrkens. Drafting the article: Lutz, Thon. Critically revising the article: Thon, Tonn, Linn, Mehrkens. Approved the final version of the manuscript on behalf of all authors: Lutz. Statistical analysis: Stahl. Administrative/technical/ material support: Lummel, Tonn.

\section{Correspondence}

Juergen Lutz, Department of Neuroradiology, Ludwig-Maximilians University, Campus Grosshadern, Marchioninistr. 15, Munich 81377, Germany.email: juergen.lutz@med.uni-muenchen.de. 